\title{
Electrical Arcing at Electrodes
}

National Cancer Institute

\section{Source}

National Cancer Institute. Electrical Arcing at Electrodes. NCI Thesaurus. Code C63303.

Problem associated with electrical current flowing through a gap between electrodes

(conductive surfaces), typically resulting in a visible flash of light. 